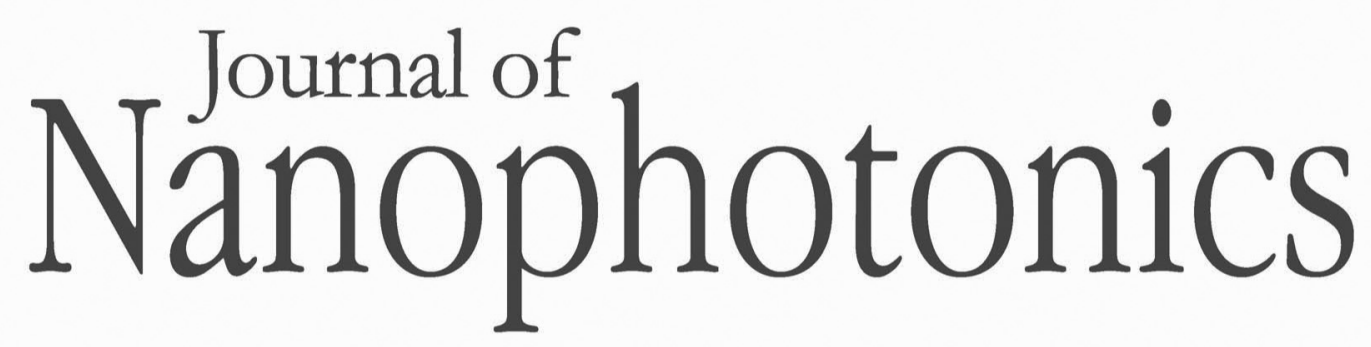

\title{
Confocal laser scanning microscopy measurement of the morphology of vanadium pentoxide nanorods grown by electron beam irradiation or thermal oxidation
}

Manil Kang

Donghyuk Hong

Taesung Kim

Minwoo Chu

Sok Won Kim 


\title{
Confocal laser scanning microscopy measurement of the morphology of vanadium pentoxide nanorods grown by electron beam irradiation or thermal oxidation
}

\author{
Manil Kang, Donghyuk Hong, Taesung Kim, Minwoo Chu, and \\ Sok Won Kim \\ University of Ulsan, Department of Physics, Ulsan 680-749, Republic of Korea \\ sokkim@ulsan.ac.kr
}

\begin{abstract}
In order to observe the morphology of nanostructures at the submicroscale, we use a confocal laser scanning (CLS) microscope built in our laboratory. The theoretical resolution of the hand-made CLS microscope is $150 \mathrm{~nm}$ and the performance of the microscope is evaluated by observing a USAF target. Vanadium pentoxide nanorods grown by electron beam irradiation and thermal oxidation methods are used as nanostructures and the morphologies of the nanorods observed by confocal laser scanning microscopy (CLSM) are compared with those obtained by scanning electron microscopy. The magnification and resolution of the CLSM were estimated to be approximately 1500 and $800 \mathrm{~nm}$, respectively. From the results, we confirm that the CLSM can be used to measure nanostructures at the sub-micro-scale without a preconditioning process. (C) The Authors. Published by SPIE under a Creative Commons Attribution 3.0 Unported License. Distribution or reproduction of this work in whole or in part requires full attribution of the original publication, including its DOI. [DOI: 10.1117/1.JNP.7.073797]
\end{abstract}

Keywords: confocal microscope; confocal laser scanning microscopy; $\mathrm{V}_{2} \mathrm{O}_{5}$; nanorods.

Paper 13043SSP received Jun. 17, 2013; revised manuscript received Aug. 21, 2013; accepted for publication Aug. 21, 2013; published online Sep. 11, 2013.

\section{Introduction}

Vanadium pentoxide $\left(\mathrm{V}_{2} \mathrm{O}_{5}\right)$ is the most stable compound in the $\mathrm{V}-\mathrm{O}$ system and exhibits highly anisotropic electrical and optical properties due to its orthorhombic structure. ${ }^{1}$ Because of its outstanding chemical, electronic, and thermal properties, ${ }^{2-5}$ nanostructured $-\mathrm{V}_{2} \mathrm{O}_{5}$ materials such as nanowires, nanorods, and nanocrystals are promising materials for application in electronic and optical devices. ${ }^{6,7}$ Several methods, including scanning electron microscopy (SEM), transmission electron microscopy (TEM), and atomic force microscopy (AFM), have been used to determine the surface morphologies of nanostructures grown by various methods. SEM is the most common method used to observe nanoscale structures with high resolution, but this method has some disadvantages. SEM requires a preconditioning process to enhance the electrical conductivity of the sample. During this process, the sample is contaminated by conductive materials such as $\mathrm{C}$ and $\mathrm{Pt}$, and can therefore not be reused for other measurements. Moreover, SEM, TEM, and AFM need to be performed by an expert operator. A nondestructive, simple method for observing the surface morphologies of nanostructures is therefore required.

We suggest the use of a confocal laser scanning (CLS) microscope to observe the sub-microscale morphology of nanostructures. ${ }^{8,9}$ A CLS microscope can provide optically sectioned images with a high lateral resolution, ${ }^{10}$ and optical three-dimensional images without physical sectioning. ${ }^{11}$ Although CLSM has a poor resolution of about $300 \mathrm{~nm}$ because of diffraction limits, ${ }^{12}$ it has several advantages compared with nonoptical methods such as SEM, TEM, and AFM. For example, because the CLSM is an optical method that involves the use of a laser, no preconditioning process is required and the sample can therefore be reused. Furthermore, measurements are easier and can be acquired in a shorter time than with the methods described above. 


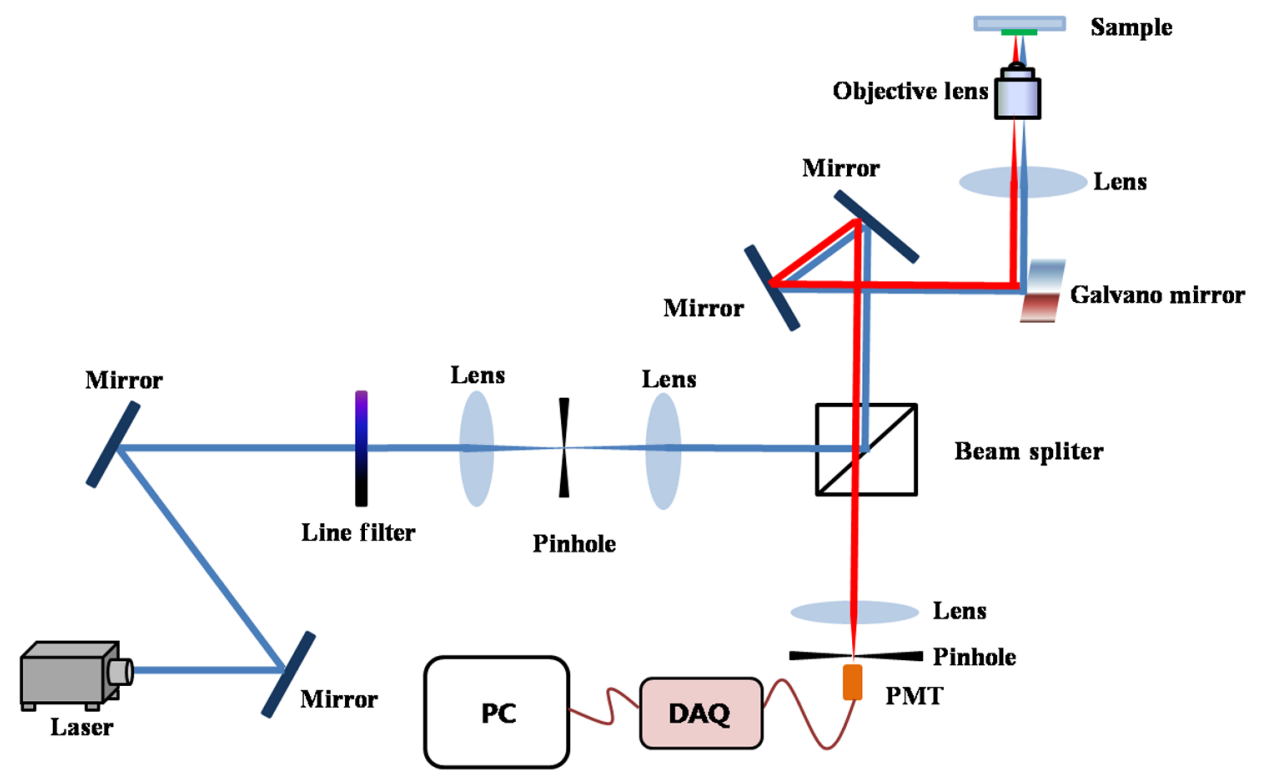

Fig. 1 Schematic diagram of our tailor-made confocal laser scanning (CLS) microscope.

The aim of this paper is to use a CLS microscope to observe the morphology of nanostructured films at the sub-micro-scale. In this study, we observed the surface morphology of $\mathrm{V}_{2} \mathrm{O}_{5}$ nanorods at the sub-micro-scale using a tailor-made CLS microscope. The resolution of the CLS microscope was evaluated by observing a USAF target, and the measurements obtained by CLSM were compared with those obtained by SEM.

\section{Experimental Details}

\subsection{Composition of the CLS Microscope}

A schematic of the microscope that we constructed in our laboratory is shown in Fig. 1. We used an Ar-ion laser (Stellar-PRO, Modu-Laser) as a light source. Because this laser emits several wavelengths of light, we inserted a line filter to remove all wavelengths except $488 \mathrm{~nm}$. The mirrors in front of the laser and the galvano mirror (MicroMax ${ }^{\mathrm{TM}}$ series 671, Cambridge Technology, Massachussetts) were used for convenient alignment of the optical components. The laser beam travels through the line filter and pinhole, which converts the beam into a point-like light source. The laser beam is then reflected by a beam splitter and meets the galvano mirror, which transforms the laser beam into a two-dimensional scan beam. This beam enters the objective lens (UPlanSApo, Olympus) with a magnification of 100× and a numerical aperture (NA) of 1.4 before reaching the sample. The laser beam reflected from the sample travels back to the galvano mirror through the objective lens, and then the beam is reflected by the galvano mirror and two additional mirrors in series, then is incident at the photo-multiplier tube (PMT, H7827-02, Hamamatsu Inc., Iwata, Japan) via a beam splitter, lens, and pinhole. The second pinhole in front of the PMT removes the out-of-focus light coming from the sample. The analog signal detected by the PMT is converted into a digital signal by data acquisition (DAQ), and the converted signal is realized as an image using Labview software.

\subsection{Preparation of Sample}

The cross-sectional structures of the $\mathrm{V}_{2} \mathrm{O}_{5}$ films deposited to grow the $\mathrm{V}_{2} \mathrm{O}_{5}$ nanorods are shown in Fig. 2. Amorphous $\mathrm{V}_{2} \mathrm{O}_{5}$ film grown on the inserted buffer layer, which is crystalline $\mathrm{V}_{2} \mathrm{O}_{5}$ grown on an $\mathrm{Al}_{2} \mathrm{O}_{3}\left(\begin{array}{llll}0 & 0 & 0 & 1\end{array}\right)$ substrate, is shown in Fig. 2(a), and amorphous $\mathrm{V}_{2} \mathrm{O}_{5}$ film grown on the substrate is shown in Fig. 2(b). A schematic diagram of a cross-section of the crystalline 

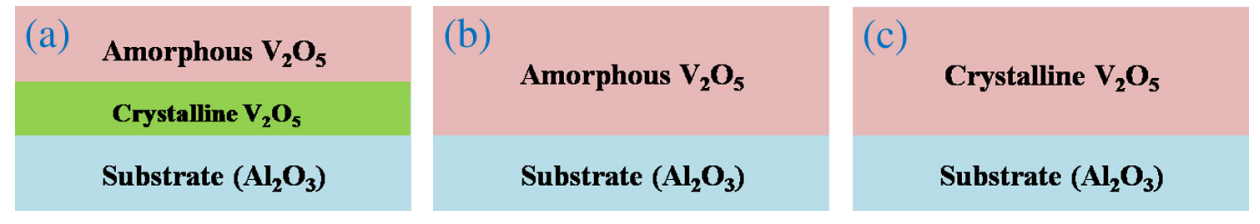

Fig. 2 Cross-sectional structures of samples: (a) amorphous $\mathrm{V}_{2} \mathrm{O}_{5}$ film inserted as a buffer layer, (b) as-grown amorphous $\mathrm{V}_{2} \mathrm{O}_{5}$ film, and (c) as-grown crystalline $\mathrm{V}_{2} \mathrm{O}_{5}$.

Table 1 Conditions and methods for growing $\mathrm{V}_{2} \mathrm{O}_{5}$ nanorods.

\begin{tabular}{lcccc}
\hline \hline Samples & Structures & $\begin{array}{c}\text { Substrate } \\
\text { temperature }\left({ }^{\circ} \mathrm{C}\right)\end{array}$ & $\begin{array}{c}\text { Film thickness } \\
(\mathrm{nm})\end{array}$ & Growing method \\
\hline 1 & Amorphous/buffer layer/substrate & $\mathrm{RT} / 500$ & $200 / 150$ & Electron beam \\
2 & Amorphous/substrate & $\mathrm{RT}$ & 200 & Electron beam \\
3 & Crystalline/substrate & 500 & 150 & Thermal oxidation \\
\hline \hline
\end{tabular}

$\mathrm{V}_{2} \mathrm{O}_{5}$ film is shown in Fig. 2(c). $\mathrm{V}_{2} \mathrm{O}_{5}$ films with various structures were prepared using an RF sputtering system with a $\mathrm{V}_{2} \mathrm{O}_{5}(99.99 \%)$ disk target having a diameter of $10 \mathrm{~cm}$, and sputtering was performed at an RF power of $200 \mathrm{~W}$. The amorphous layer was grown at room temperature for $200 \mathrm{~min}$, and crystalline layers were deposited at a substrate temperature of $500^{\circ} \mathrm{C}$ for 150 min. Ar and $\mathrm{O}_{2}$ gas, each with a purity of $99.999 \%$, were used as the sputtering gas and reactive gas, respectively.

The thicknesses of the films were measured by a spectroscopic ellipsometer (Jobin-Yvon, Uvisel UV/NIR) and the thickness of the amorphous and crystalline layers were found to be 200 and $150 \mathrm{~nm}$, respectively. The conditions used to deposit the $\mathrm{V}_{2} \mathrm{O}_{5}$ films and the method used to grow $\mathrm{V}_{2} \mathrm{O}_{5}$ nanorods are listed in Table 1 .

We used electron beam irradiation to grow the $\mathrm{V}_{2} \mathrm{O}_{5}$ nanorods. Two kinds of $\mathrm{V}_{2} \mathrm{O}_{5}$ films [see Figs. 2(a) and 2(b)] were irradiated by an electron beam with an energy of $0.7 \mathrm{MeV}$ using an electron beam accelerator (BINP, ELV-0.5). Samples 1 and 2 were irradiated at dose rates of $1000 \mathrm{kGy}$ for $90 \mathrm{~s}$ and $1200 \mathrm{kGy}$ for $105 \mathrm{~s}$, respectively. We also used a thermal oxidation method to grow $\mathrm{V}_{2} \mathrm{O}_{5}$ nanorods. Sample 3 was postannealed at a temperature of $650^{\circ} \mathrm{C}$ for $30 \mathrm{~min}$ in ambient $\mathrm{O}_{2}$ in a furnace.

\section{Results and Discussion}

The resolution of an optical microscope can be calculated using Eq. (1): ${ }^{13}$

$$
r_{\text {lateral }}=\frac{0.61 \lambda}{\mathrm{NA}}=\frac{1.22 \lambda}{2 \mathrm{NA}}
$$

where $r_{\text {lateral }}$ is the lateral resolution, $\lambda$ is the wavelength of light, and NA is the numerical aperture of the objective lens. However, the full-width-at-half-maximum of a confocal microscope is narrower than that of a conventional microscope. So, the theoretical $r_{\text {lateral }}$ of the confocal microscope should be calculated as shown in Eq. (2): ${ }^{14}$

$$
r_{\text {lateral }}=\frac{0.61 \lambda}{\mathrm{NA} \sqrt{2}}=\frac{0.4 \lambda}{\mathrm{NA}} .
$$

The theoretical resolution of the CLS microscope used in this study was found to be $150 \mathrm{~nm}$. However, because of several factors, such as aberrations introduced by the optical components, 

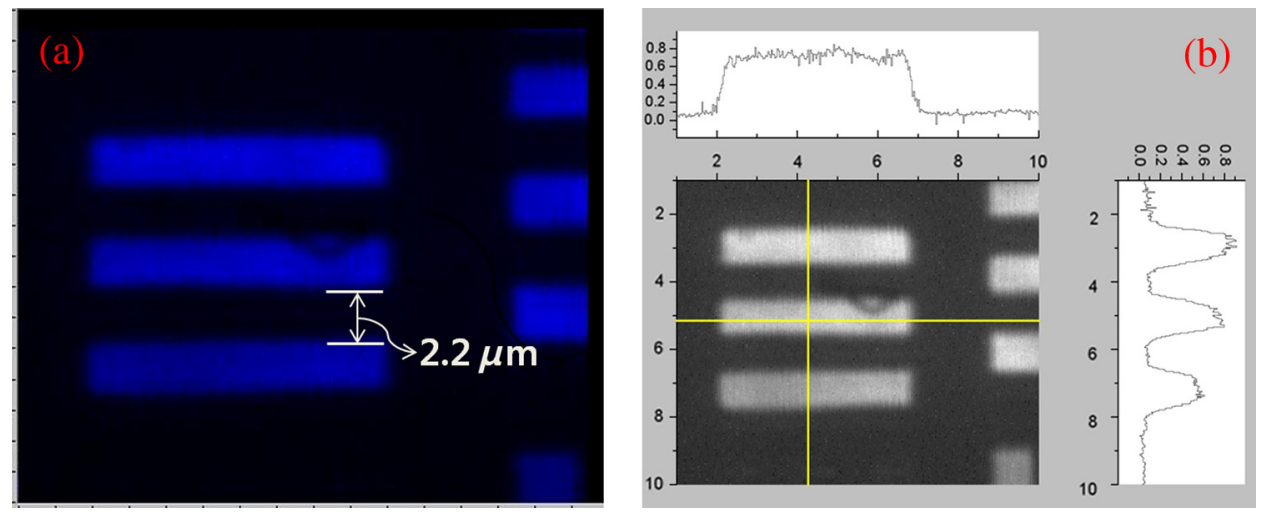

Fig. 3 (a) Image of USAF 1951 target measured by our confocal laser scanning microscope; the distance between bars was $2.2 \mu \mathrm{m}$. (b) An intensity profile of the image shown in Fig. 3(a).

misalignment, and scattering from the nanorods, the practical resolution was expected to be lower than the theoretical resolution.

To evaluate the resolution of our microscope, we used a USAF 1951 target. Figure 3(a) shows the group 7-element 6, which is the smallest section in the USAF 1951 target. As shown in Fig. 3 (a), a clear image was obtained, and the distance between bars was confirmed to be $2.2 \mu \mathrm{m}$. An intensity profile of the image in Fig. 3(a) is provided in Fig. 3(b). The profile revealed a distribution of stable intensity.

The surface morphology of the $\mathrm{V}_{2} \mathrm{O}_{5}$ nanorods grown by irradiation of $\mathrm{V}_{2} \mathrm{O}_{5}$ film with an electron beam at a dose rate of $1000 \mathrm{kGy}$ is shown in Fig. 4 (Sample 1). The morphology of the $\mathrm{V}_{2} \mathrm{O}_{5}$ nanorods measured by SEM (JEOL, JSM6335F) at a magnification of 20,000× is shown in Fig. 4(a). Well-grown $\mathrm{V}_{2} \mathrm{O}_{5}$ nanorods were clearly observed. The width and length of the nanorods were confirmed to be approximately 150 to $300 \mathrm{~nm}$ and 1 to $2 \mu \mathrm{m}$, respectively. Figure 4(b) shows the morphology of the $\mathrm{V}_{2} \mathrm{O}_{5}$ nanorods obtained by our CLS microscope at a pixel resolution of $309 \times 309$. As shown in Fig. 4(b), although the shape of the nanorods could be distinguished, a clear image was not obtained and the width and length of the nanorods could also not be determined. This result is due to the resolution limit of our microscope. The practical resolution of our microscope was lower than the theoretically predicted value because of factors such as aberrations introduced by the optical components, misalignment, and scattering from the nanorods.
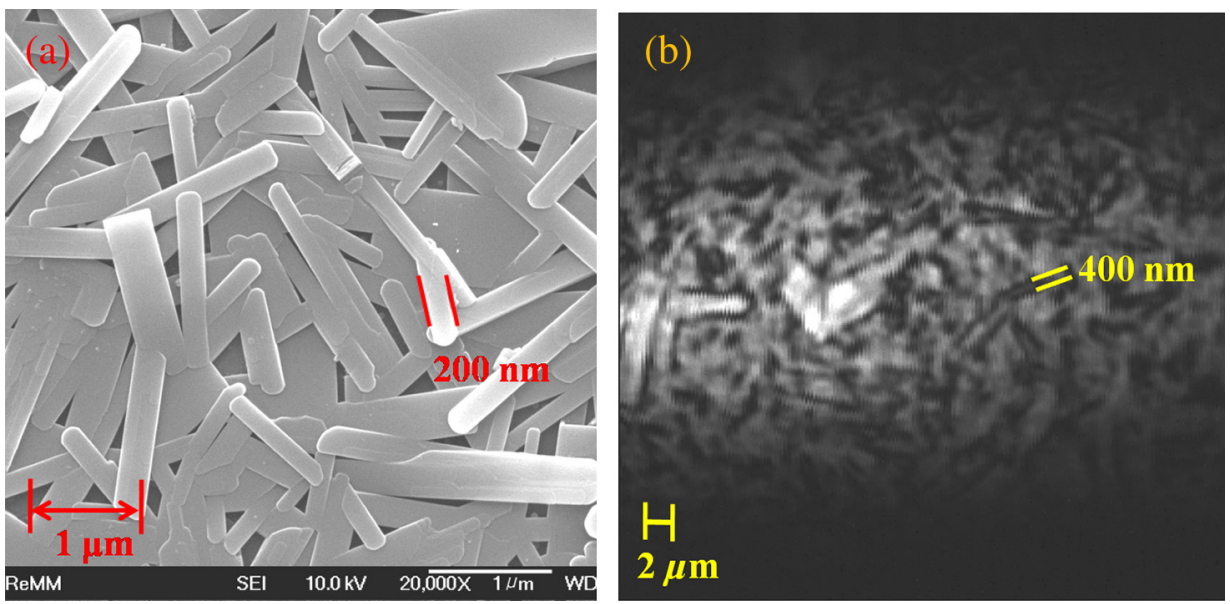

Fig. 4 Surface morphology of $\mathrm{V}_{2} \mathrm{O}_{5}$ nanorods grown by electron beam irradiation of $\mathrm{V}_{2} \mathrm{O}_{5}$ film at a dose rate of $1000 \mathrm{kGy}$. The morphologies obtained by (a) SEM $(20,000 \times)$ and (b) CLSM $(309 \times 309$ pixels $)$. 

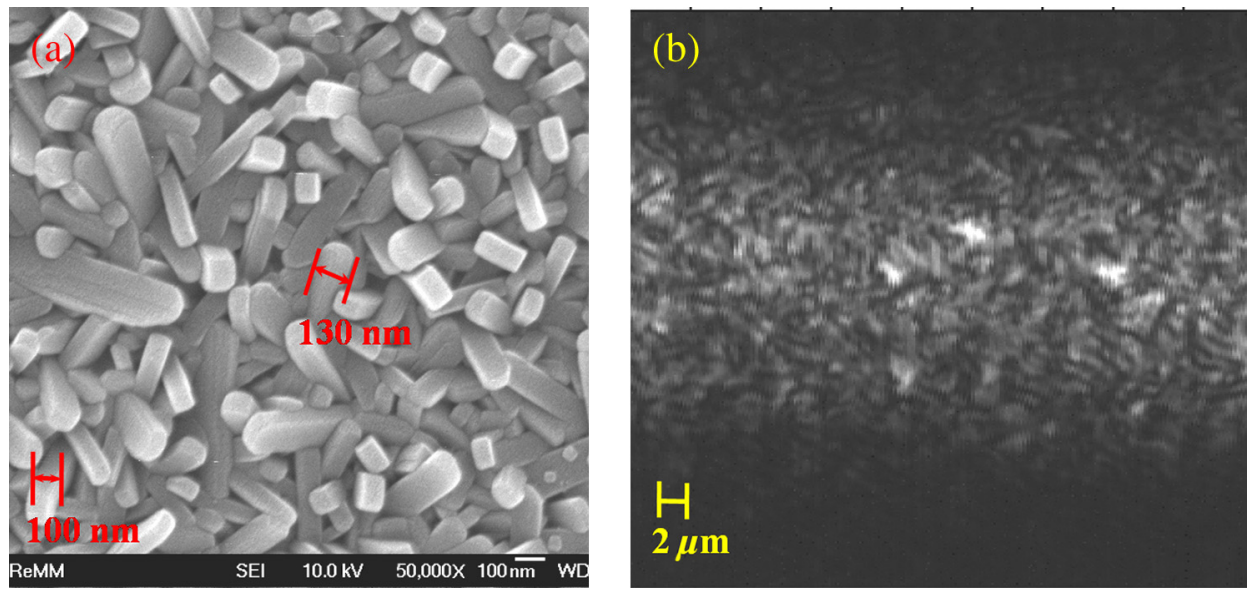

Fig. 5 Surface morphology of $\mathrm{V}_{2} \mathrm{O}_{5}$ nanorods grown by irradiating $\mathrm{V}_{2} \mathrm{O}_{5}$ film with an electron beam at a dose rate of $1200 \mathrm{kGy}$ (Sample 2). Morphologies obtained by (a) SEM $(50,000 \times)$ and (b) CLSM $(309 \times 309$ pixels).

Figure 5 shows the surface morphology of $\mathrm{V}_{2} \mathrm{O}_{5}$ nanorods grown by irradiating $\mathrm{V}_{2} \mathrm{O}_{5}$ film with an electron beam at a dose rate of $1200 \mathrm{kGy}$ (Sample 2). Figure 5(a) shows the morphology of $\mathrm{V}_{2} \mathrm{O}_{5}$ nanorods obtained by SEM at a magnification of 50,000. Densely grown $\mathrm{V}_{2} \mathrm{O}_{5}$ nanorods with a width of $\sim 130 \mathrm{~nm}$ were observed. The morphology of the $\mathrm{V}_{2} \mathrm{O}_{5}$ nanorods could not be distinguished in the CLSM image shown in Fig. 5(b). We attributed this result to the resolution limit of our CLS microscope.

The surface morphology of $\mathrm{V}_{2} \mathrm{O}_{5}$ nanorods grown by the thermal oxidation method is shown in Fig. 6. $\mathrm{V}_{2} \mathrm{O}_{5}$ film was postannealed in a furnace at a temperature of $650^{\circ} \mathrm{C}$ for $30 \mathrm{~min}$ in ambient $\mathrm{O}_{2}$ (Sample 3). As shown in Fig. 6(a), the morphology obtained by SEM at a magnification of $1500 \times$ showed the growth of $\mathrm{V}_{2} \mathrm{O}_{5}$ rods with a width of $\sim 1 \mu \mathrm{m}$ and a length of $\sim 10 \mu \mathrm{m}$. The morphology obtained by CLSM, shown in Fig. 6(b), is similar to that obtained by SEM. Well-grown $\mathrm{V}_{2} \mathrm{O}_{5}$ nanorods were clearly observed, and the width of the nanorods was confirmed to be approximately $800 \mathrm{~nm}$. This result indicates that the CLS microscope in this study can be used to measure the morphologies of nanostructures at the sub-micro-scale. By comparing these results with those obtained by SEM, we inferred that the magnification and resolution of our CLS microscope were above approximately 1500 and below approximately $800 \mathrm{~nm}$, respectively.
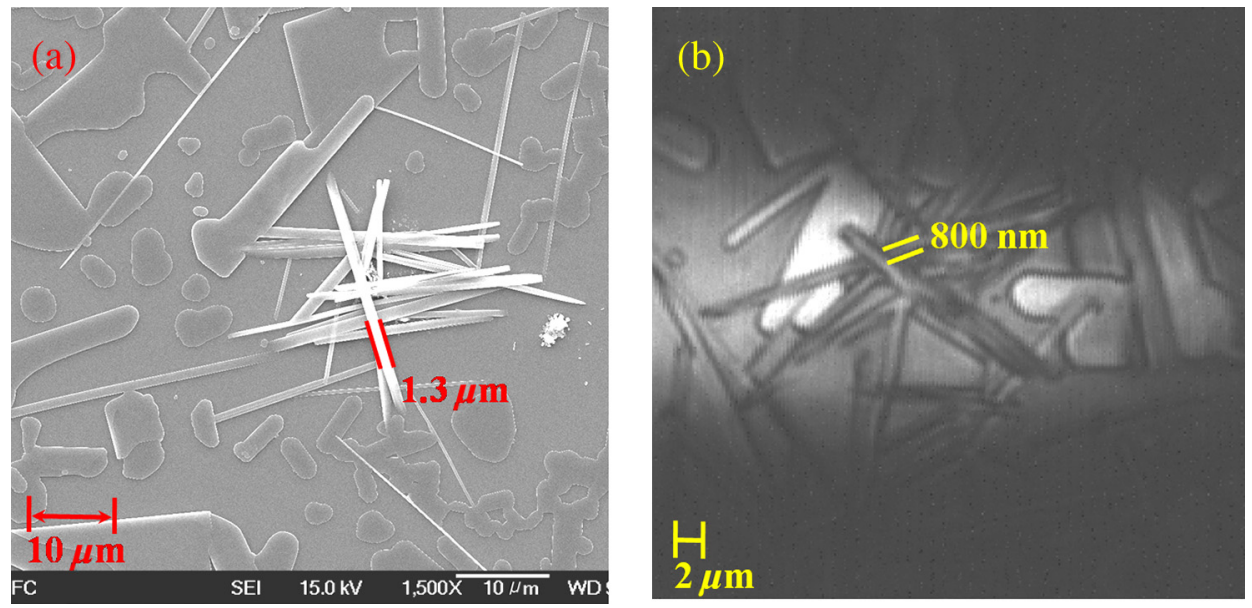

Fig. 6 Surface morphology of $\mathrm{V}_{2} \mathrm{O}_{5}$ nanorods grown by thermal oxidation. The morphologies obtained by (a) SEM (1500 $\times)$ and (b) CLSM $(309 \times 309$ pixels). 


\section{Conclusions}

We proposed using CLSM to observe the morphology of nanostructures at the sub-micro-scale. We examined $\mathrm{V}_{2} \mathrm{O}_{5}$ nanorods grown by electron beam irradiation and thermal oxidation using a confocal scanning laser microscope that we built in our laboratory. The theoretical resolution of the hand-made CLSM was found to be $150 \mathrm{~nm}$ and was evaluated by observing a USAF target.

The morphologies of the $\mathrm{V}_{2} \mathrm{O}_{5}$ nanorods grown by the electron beam irradiation and thermal oxidation methods were observed by CLSM and compared with those obtained by SEM. By comparing the CLSM results with the SEM results, we estimated that the magnification and resolution of our confocal scanning laser microscope were above approximately $1500 \mathrm{~nm}$ and below approximately $800 \mathrm{~nm}$, respectively. These results indicate that the CLSM can be used to measure the morphologies of nanostructures at the sub-micro-scale, and is a nondestructive, simple method without a preconditioning process compared with other tools such as SEM, TEM, and AFM.

\section{Acknowledgments}

This work was supported by the Priority Research Centers Program through the National Research Foundation of Korea (NRF) funded by the Ministry of Education, Science, and Technology (2009-0093818) and by the development program of the local science park funded by the ULSAN Metropolitan City and the Ministry of Education, Science, and Technology. Donghyuk Hong and Taesung Kim built the CLS microscope and obtained the sample images, Manil Kang and Minwoo Chu prepared the samples and wrote this paper, and Sok Won Kim supervised this investigation.

\section{References}

1. L. R. Smith et al., "A scanning probe microscopy study of the $\left(\begin{array}{lll}0 & 0 & 1\end{array}\right)$ surfaces of $\mathrm{V}_{2} \mathrm{O}_{5}$ and $\mathrm{V}_{6} \mathrm{O}_{13}$," Surf. Sci. 367(1), 87-95 (1996), http://dx.doi.org/10.1016/S0039-6028(96) 00858-8.

2. Y. Wang and G. Cao, "Synthesis and enhanced intercalation properties of nanostructured vanadium oxides," Chem. Mater. 18(12), 2787-2804 (2006), http://dx.doi.org/10.1021/ cm052765h.

3. V. Petkov et al., "Structure beyond Bragg: study of $\mathrm{V}_{2} \mathrm{O}_{5}$ nanotubes," Phys. Rev. B 69(8), 085410 (2004), http://dx.doi.org/10.1103/PhysRevB.69.085410.

4. G. T. Kim et al., "Field-effect transistor made of individual $\mathrm{V}_{2} \mathrm{O}_{5}$ nanofibers," Appl. Phys. Lett. 76(14), 1875-1877 (2000), http://dx.doi.org/10.1063/1.126197.

5. C. V. Ramana et al., "Correlation between growth conditions, microstructure, and optical properties in pulsed-laser-deposited $\mathrm{V}_{2} \mathrm{O}_{5}$ thin films," Chem. Mater. 17(5), 1213-1219 (2005), http://dx.doi.org/10.1021/cm048507m.

6. C. G. Granqvist, Handbook of Inorganic Electrochromic Materials, Elsevier, Amsterdam (1995).

7. B. B. Lakshmi, C. J. Patrissi, and C. R. Martin, "Sol-gel template synthesis of semiconductor oxide micro- and nanostructures," Chem. Mater. 9(11), 2544-2550 (1997), http://dx .doi.org/10.1021/cm970268y.

8. C. W. Zou et al., "Enhanced visible photoluminescence of $\mathrm{V}_{2} \mathrm{O}_{5}$ via coupling $\mathrm{ZnO} / \mathrm{V}_{2} \mathrm{O}_{5}$ composite nanostructures," Opt. Lett. 35(8), 1145-1147 (2010), http://dx.doi.org/10.1364/ OL.35.001145.

9. J. T. Fredrich, "3D imaging of porous media using laser scanning confocal microscopy with application to microscale transport process," Phys. Chem. Earth A 24(7), 551-561 (1999), http://dx.doi.org/10.1016/S1464-1895(99)00079-4.

10. B. R. Boruah and M. A. A. Neil, "Programmable diffractive optics for laser scanning confocal microscopy,” Proc. SPIE 6443, 644310 (2007), http://dx.doi.org/10.1117/12.700611.

11. M. Müller, Introduction to Confocal Fluorescence Microscopy, SPIE Press, Bellingham (2006). 
12. J. F. van Derlofske, "Computer modeling of LED light pipe systems for uniform display illumination," Proc. SPIE 4445, 119-129 (2001), http://dx.doi.org/10.1117/12.450035.

13. T. J. Kim, D. G. Gweon, and H. H. Lee, "Enhancement of fluorescence confocal scanning microscopy lateral resolution by use of structured illumination," Meas. Sci. Technol. 20(5), 055501 (2009), http://dx.doi.org/10.1088/0957-0233/20/5/055501.

14. J. B. Pawley, Handbook of Biological Confocal Microscopy, 3rd ed., Springer, Wisconsin (2006).

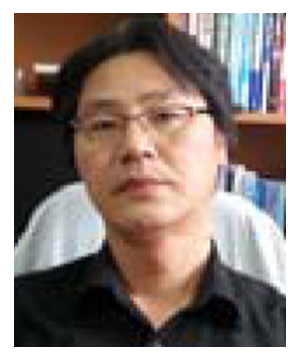

Manil Kang's major is thin film optics, and he is a research professor at the Basic Science Research Institute at the University of Ulsan. His main research fields are thin film optics, ellipsometry, and strong correlation, and he is currently investigating nanostructured metal oxides and phase transitions in vanadium oxides. He has published more than 10 research papers in APL, AIP Advances, Thin Solid Films, Thermochimica Acta, etc.

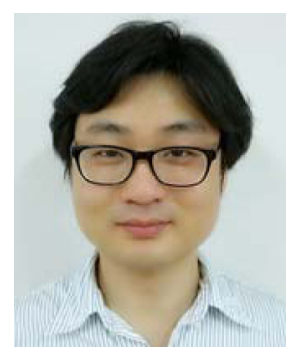

Donghyuk Hong is pursuing his doctorate in the Department of Physics at the University of Ulsan and is investigating confocal laser scanning microscopy. This year, he published a research paper, "Development of a low-cost microscope using a DVD optical pickup head," in Optik.

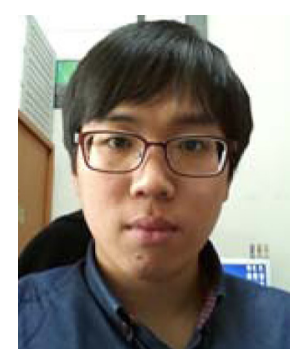

Taesung Kim is an undergraduate in the Department of Physics at the University of Ulsan and is investigating confocal laser scanning microscopy. He has participated in conferences held by the Korean Physical Society and the Korean Optical Society.

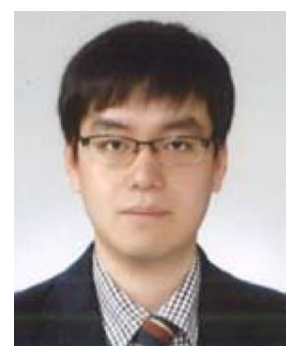

Minwoo Chu took his master's course in the Department of Physics at the University of Ulsan and is investigating vanadium oxides. This year, he published a research paper, "Optical and electrical properties of $\mathrm{V}_{2} \mathrm{O}_{5}$ nanorod films using electron beam," in Thin Solid Films.

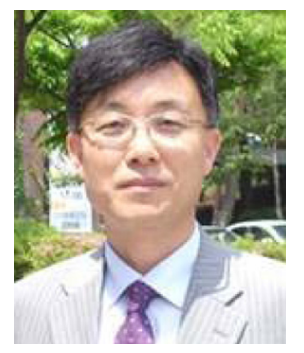

Sok Won Kim's major is laser optics, and he is a professor in the Department of Physics at the University of Ulsan. His main research fields are confocal laser scanning microscopy, fluorescence correlation spectroscopy, thermal physics, and material optics, and he is currently investigating carbon composite materials and vanadium oxides. He has published more than 30 research papers in APL, AIP Advances, Thin Solid Films, Thermochimica Acta, etc. 